Neural Networks and Learning Systems for Human Machine Interfacing

Dr. Zhaojie Ju, Prof. Jinguo Liu, Prof. YongAn Huang , Prof. Naoyuki Kubota, Prof. John Q. Gan

PII:

DOI:

Reference:

To appear in:

Received date:

Accepted date:
S0925-2312(19)31447-X

https://doi.org/10.1016/j.neucom.2019.10.058

NEUCOM 21414

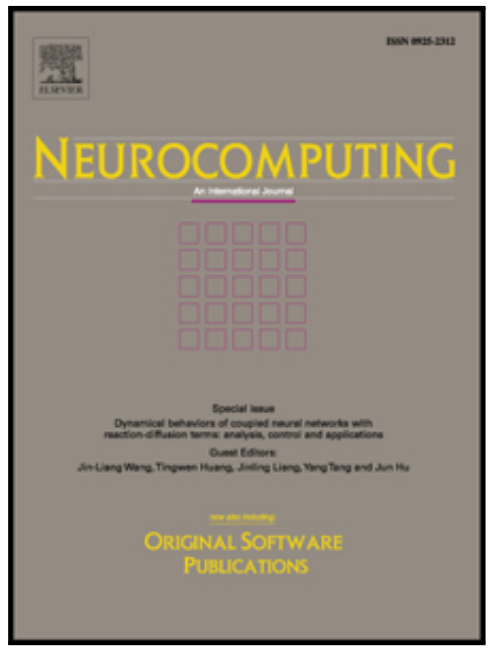

Please cite this article as: Dr. Zhaojie Ju, Prof. Jinguo Liu, Prof. YongAn Huang, Prof. Naoyuki Kubota, Prof. John Q. Gan, Neural Networks and Learning Systems for Human Machine Interfacing, Neurocomputing (2019), doi: https://doi.org/10.1016/j.neucom.2019.10.058

This is a PDF file of an article that has undergone enhancements after acceptance, such as the addition of a cover page and metadata, and formatting for readability, but it is not yet the definitive version of record. This version will undergo additional copyediting, typesetting and review before it is published in its final form, but we are providing this version to give early visibility of the article. Please note that, during the production process, errors may be discovered which could affect the content, and all legal disclaimers that apply to the journal pertain.

(C) 2019 Published by Elsevier B.V. 
Editorial

\section{Neural Networks and Learning Systems for Human Machine Interfacing}

With developments of the sensor and computing technologies, human-machine interfaces (HMIs) are designed to meet the increasing user demands of machines and systems. This is because human effects are becoming the key issues to allow some advanced mechanical devices, such as robots and biometric systems, to perform complicate tasks intelligently in an unknown environment. An effective $\mathrm{HMl}$ with learning ability can process, interpret, recognize, and simulate the intention and behaviors of human beings, and then utilize intelligent algorithms to drive the machine devices. The HMls also enable us to bring humanistic intelligence and actions in robotic devices, biometric systems and other machines through two-ways interactions, such as using deep neural networks. In recent years, a growing number of researchers and studies focusing on this area have clearly demonstrated the importance of learning systems for HMls.

This special issue provides a forum to discuss not only developments and research progresses in HMls, but also challenges in the research and development of neural networks and learning systems for HMls. This special issue attracted a total of 38 paper submissions, spanning over numerous active and emerging topic areas. After a rigorous review process, 9 high-quality papers are accepted and published in this special issue.

The paper titled "Robust real-time hand detection and localization for space human-robot interaction based on deep learning" proposes a feature-map-fused single shot multibox detector (FF-SSD) for robust real-time hand detection and localization. The proposed FF-SSD method fuses a shallow layer with a deep layer, and this can increase the detection accuracy of pony-size hands. Finally, this method has been implemented in space human-robot interaction.

The paper titled "Counting crowds using a scale-distribution-aware network and adaptive human-shaped kernel" develops a crowd counting algorithm to provide the counting information for a bus dispatch system in a human-machine system, in which a scale-distribution-aware multi-column convolutional neural network (SDA-MCNN) is developed to count crowds by summing up the output (denoted as the density map) of the SDA-MCNN.

The paper titled "Improved Itracker Combined with Bidirectional Long Short-Term Memory for 3D Gaze Estimation using Appearance Cues" proposes an improved Itracker to predict the subject's gaze for a single image frame, as well as employs a many-to-one bidirectional Long Short-Term Memory to fit the temporal information between frames to better estimate gaze for video sequences.

The paper titled "Classifying ASD Children with LSTM based on Raw Videos" proposes to use deep learning to help diagnose ASD in raw video data. This method tracks the eye movement in each video by the tracking-learning-detection method and 
adopts three-layer Long Short-Term Memory network for classification.

The paper titled "Text-based indoor place recognition with deep neural network" presents a new indoor place recognition scheme using deep neural network to improve the recognition framework by utilizing Place Descriptors in text from to connect different types of place information with their categories.

The paper titled "Visual Recognition of Traffic Police Gestures with Convolutional Pose Machine and Handcrafted Features" proposes a novel vision-based human-machine interface to recognize eight kinds of Chinese traffic police gestures and apply it in the real-time recognition tasks. This method integrates a modified CPM network and two kinds of handcrafted features.

The paper titled "A Robot Learning Framework based on Adaptive Admittance Control and Generalizable Motion Modeling with Neural Network Controller" developed a neural network-based controller for the robot to track the trajectories generated from the motion model, in which a radial basis function neural network is designed to compensate for the effect caused by the dynamic environments.

The paper titled "Estimation of Human Impedance and Motion Intention for Constrained Human-Robot Interaction" proposes an adaptive neural network impedance control involving full-state constraints in human robot collaborative tasks, which employs radial basis function neural networks to estimate the human motion intention in real time, and the least square method is utilized in robot learning of human impedance.

The paper titled "A learning-based multiscale modelling approach to real-time serial manipulator kinematics simulation" proposes a novel learning-based multiscale modelling approach to address the efficiency and accuracy issues through a combination of models with different levels of fidelity.

\section{Acknowledgments}

We would like to thank all reviewers for their timely and insightful comments for this special issue. All the authors who submitted papers have contributed significantly to the issue by taking an exploratory role in the development of this important field of inquiry. We also would like to express our appreciation to the editor-in-chief and editorial office of the Neurocomputing (Elsevier) journal for their strong support.

\section{Authors:}

Dr. Zhaojie Ju (zhaojie.ju@port.ac.uk, University of Portsmouth, UK)

Prof. Jinguo Liu (liujinguo@sia.cn, Chinese Academy of Sciences, China)

Prof. YongAn Huang (yahuang@hust.edu.cn, Huazhong University of Science and Technology, China)

Prof. Naoyuki Kubota (kubota@tmu.ac.jp, Tokyo Metropolitan University, Japan)

Prof. John Q. Gan (jqgan@essex.ac.uk, University of Essex, UK) 


\section{Declaration of interests}

The authors dec lare that they have no known competing financial interests or personal relationships that could have appeared to influence the work reported in this paper. 